\title{
Do dietary interventions improve health outcomes in people with multiple sclerosis? A Cochrane Review summary with
} commentary

\author{
Bhasker Amatya ${ }^{\mathrm{a}, \mathrm{b}, *}$ and Fary Khan ${ }^{\mathrm{a}, \mathrm{b}}$ \\ ${ }^{a}$ Department of Rehabilitation and Australian Rehabilitation Research Centre, Royal Melbourne Hospital, \\ Parkville, VIC, Australia \\ ${ }^{\mathrm{b}}$ Department of Medicine (Royal Melbourne Hospital), the University of Melbourne, Parkville, VIC, Australia
}

\begin{abstract}
.
BACKGROUND: A broad range of complementary and alternative medicine (CAM) approaches, including different dietary interventions, alone or in conjunction with conventional medicine are currently trailed in people with multiple sclerosis (MS). Published clinical and experimental data suggest that certain dietary interventions may improve MS-related health outcomes. OBJECTIVE: To assess the effectiveness of dietary interventions to improve MS-related health outcomes.

METHODS: To summarize the updated Cochrane Review "Dietary interventions for multiple sclerosis-related outcomes" conducted by Parks et al. Best available evidence was discussed from the rehabilitation perspective.

RESULTS: Overall 30 RCTs (with 2335 participants) evaluated a range of dietary interventions: polyunsaturated fatty acids, antioxidant supplements, dietary programmes and other dietary supplements. All included trials had one or more methodological issues leading to an unknown or high risk of bias. The findings suggest that the evidence is uncertain about the effect of dietary interventions on MS-related health outcomes.

CONCLUSIONS: The evidence for dietary interventions in people with MS is sparse and uncertain, and more robust studies are needed.
\end{abstract}

Keywords: Multiple sclerosis, diet, health outcomes, rehabilitation

The aim of this commentary is to discuss the rehabilitation perspective in the published Cochrane Review "Dietary interventions for multiple sclerosisrelated outcomes" (Parks et al., 2020) by Parks NE, Jackson-Tarlton CS, Vacchi L, Merdad R, Johnston

\footnotetext{
*Address for correspondence: Dr. Bhasker Amatya, Department of Rehabilitation Medicine, Royal Melbourne Hospital, 34-54 Poplar Road, Parkville, VIC 3052, Australia. Tel.: +61 3 83872037; Fax: +61 3 83872222; E-mail: bhasker.amatya @mh.org.au.
}

$\mathrm{BC}^{\mathrm{a}}$, under the direct supervision of Cochrane Multiple Sclerosis and Rare Diseases of the CNS Group.

\footnotetext{
${ }^{\text {a }}$ This summary is based on a Cochrane Review previously published in the Cochrane Database of Systematic Reviews 2020, Issue 5, Art. No.: CD004192, DOI: 10.1002/14651858.CD004192.pub4 (see www.cochranelibrary.com for information). Cochrane Reviews are regularly updated as new evidence emerges and in response to feedback, and Cochrane Database of Systematic Reviews should be consulted for the most recent version of the review.

The views expressed in the summary with commentary are those of the Cochrane Corner authors and do not represent the Cochrane Library or Wiley.
} 
This Cochrane Corner is produced in agreement with NeuroRehabilitation by Cochrane Rehabilitation.

\section{Background}

Multiple sclerosis (MS) is an immune-mediated disease of the central nervous system causing multiple disabilities, including disorders of strength, sensation, coordination and balance, visual, and cognitive deficits (Noseworthy, et al., 2000). These impairments usually lead to progressive limitation of functioning in daily life and have significant economic implications and burden to the patients, carers/family, community and healthcare system.

MS is a complex condition requiring a longterm comprehensive and multidimensional approach to disease management, which incorporates pharmacological and non-pharmacological interventions. There is no cure for MS at present, and many patients tend to use different forms of complementary and alternative medicine (CAM) alone or in conjunction with conventional medicine, with dietary interventions being the most popular (O'Conner et al., 2012). A recent updated Cochrane Review (Parks et al., 2020) evaluated the evidence for the effectiveness of various dietary interventions used to improve health outcomes in people with MS, aiming to guide the treating clinicians.

\section{Dietary interventions for multiple sclerosis-related outcomes}

(Parks NE, Jackson-Tarlton CS, Vacchi L, Merdad R, Johnston BC. 2020)

\section{What is the aim of this Cochrane review?}

The aim of this Cochrane Review was to evaluate the effectiveness of different types of dietary interventions in improving MS-related outcomes in people with MS.

\section{What was studied and methods?}

The population was adults with clinically definite MS (all types) regardless of age, sex, duration of MS, degree of disability, course of the disease and current/prior MS therapy. The primary outcomes included: relapse and change in disability progression, while, secondary outcomes were: magnetic resonance imaging (MRI) activity, safety, and patientreported outcomes (such as health-related quality of life (HRQoL), cognitive function, fatigue, etc.). The interventions included any dietary supplements, dietary plans, specific foods, macronutrients, or natural health products (except Vitamin-D, as it was subject to separate Cochrane review). The risk of bias in included studies was assessed with the Cochrane risk-of-bias tool and the certainty of the evidence was assessed using the GRADE approach.

\section{Search methodology and up-to-dateness of the Cochrane review?}

A comprehensive search of CENTRAL, MEDLINE, Embase, and Web of Science databases and clinical trials registries up to 30 May 2019 was performed for randomized controlled trials (RCTs) and controlled-clinical trials examining the effects of any dietary intervention for MS versus placebo or another dietary intervention.

\section{What are the main results of the Cochrane review?}

The review included 30 RCTs (with 2335 participants). The dietary interventions assessed varied across the included studies and the majority evaluated polyunsaturated fatty acids (PUFAs), followed by antioxidant supplements, dietary programmes and other dietary supplements. All trials had one or more methodological issues leading to an unknown or high risk of bias.

Key findings of the review are summarized below.

- PUFAs compared to monounsaturated fatty acids

- Very low certainty evidence concerning the difference in relapses (risk ratio (RR): 1.02, 95\% confidence interval (CI): 0.88 to 1.20 ) and change in disability progression

- Low certainty evidence of little or no difference in global impression of deterioration (RR: 0.85 , 95\%CI: 0.71 to 1.03 )

- Comparison of the different PUFAs: omega-3 versus omega-6

- Low certainty evidence in little to no difference in relapses (RR: 1.02, 95\% CI: 0.62, to 1.66 ), change in disability progression, measured as change in Expanded Disability 
Status Scale (EDSS) (mean difference (MD): $0.00,95 \% \mathrm{CI}:-0.30$ to 0.30 ), and serious adverse events (RR: $1.12,95 \% \mathrm{CI}$ : 0.38 to 3.31 )

- Moderate certainty evidence of little or no difference in global impression of deterioration (RR: 0.99 , 95\%CI: 0.51 to 1.91 )

- Antioxidant supplements compared with placebo

- Low certainty evidence of little or no difference in reducing relapses (RR: 0.98, 95\% CI: 0.59 to 1.64$)$ and global impression of deterioration (RR: $0.99,95 \%$ CI: 0.50 to 1.93)

- Low certainty evidence of little or no difference in serious adverse events (RR: 0.72, 95\%CI: 0.17 to 3.08 )

- Very low certainty evidence concerning the difference in improvement in disability progression (MD: $-0.19,95 \%$ CI: -0.49 to 0.11 ) and MRI activity - gadolinium enhancing lesions (RR: 0.67, 95\%CI: 0.09 to 4.88 )

- Low certainty evidence of little or no difference in improvement in fatigue (SMD $-0.38,95 \%$ CI: -0.96 to 0.19 )

- Very uncertainty evidence in favor of dietary interventions for improvement in HRQoL, cognitive outcomes

- For the comparison of dietary programmes, review authors did not include data synthesis because of heterogeneity of intervention types (diet protocol, outcome measures) across studies

\section{How did the authors conclude on the evidence?}

The authors concluded that evidence regarding the effects of dietary interventions in people with MS is very uncertain. There is insufficient evidence to determine whether PUFA, antioxidant supplementation, dietary programmes, and other natural health product supplementation have an effect on MS-related outcomes such as relapses, disability progression and global impression of deterioration. There is a need for more robust RCTs examining the effectiveness of these interventions in the MS population.

\section{What are the implications of the Cochrane evidence for practice in rehabilitation?}

The findings of this review do not support the prescription of PUFAs, antioxidant supplements, dietary programmes and other dietary supplements, as the certainty of the evidence of the effect estimates for both benefit and harm outcomes is low or very low.

\section{Acknowledgments}

The authors thank Cochrane Rehabilitation and Cochrane Multiple Sclerosis and Rare Diseases of the CNS Group for reviewing the contents of the Cochrane Corner.

\section{Conflict of interest}

The authors declare no conflicts of interest.

\section{References}

Noseworthy, J. H., Lucchinetti, C., Rodriguez, M., \& Weinshenker, B. G. (2000) Multiple sclerosis. N Engl J Med, 343, 938-952. DOI: 10.1056/NEJM200009283431307

Parks, N. E., Jackson-Tarlton, C. S., Vacchi, L., Merdad, R., \& Johnston, B. C. (2020) Dietary interventions for multiple sclerosis-related outcomes. Cochrane Database Syst Rev (5). Art. No.: CD004192. DOI: 10.1002/14651858. CD004192.pub4

O'Connor, K., Weinstock-Guttman, B., Carl, E., Kilanowski, C., Zivadinov, R., \& Ramanathan, M. (2012) Patterns of dietary and herbal supplement use by multiple sclerosis patients. J Neurol, 259, 637-644. DOI: 10.1007/s00415-011-6226-3 